Portland State University

PDXScholar

8-1-1967

\title{
Landscape and figure composition in relation to space, color, and line
}

Perry Theo Carandanis

Portland State University

Follow this and additional works at: https://pdxscholar.library.pdx.edu/open_access_etds Let us know how access to this document benefits you.

Recommended Citation

Carandanis, Perry Theo, "Landscape and figure composition in relation to space, color, and line" (1967). Dissertations and Theses. Paper 485.

https://doi.org/10.15760/etd.485

This Thesis is brought to you for free and open access. It has been accepted for inclusion in Dissertations and Theses by an authorized administrator of PDXScholar. Please contact us if we can make this document more accessible: pdxscholar@pdx.edu. 


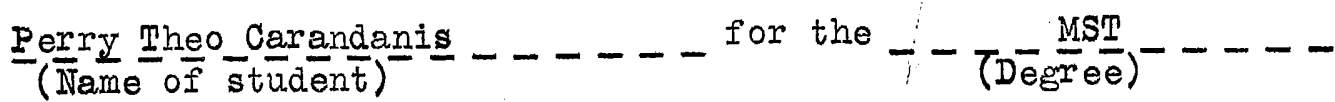


Title:_ _ _ Iandscape and Figure Composition in Relation to

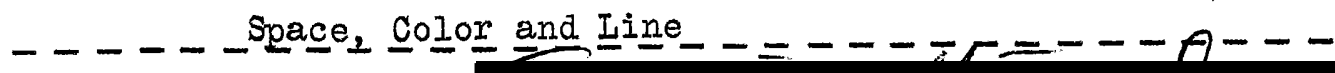
Abstract Approved: (Signature)

Frederick Heidel

The thesis is an empirical analysis of eight paintings in which the problems to be examined are the relationships of the expressive visual elements of composition with the subject matter. An attempt will be made to examine some specific problems encountered with individual works, which will include emotional involvement as well as aesthetic, and arrive at some generalizations. The paintings selected to comprise this thesis are four figure compositions and three landscapes. The medium used to execute the paintings is Acrylic-Polymer, a Hyplar Product, and they are painted on Upsom Board, for which considerations are given. 

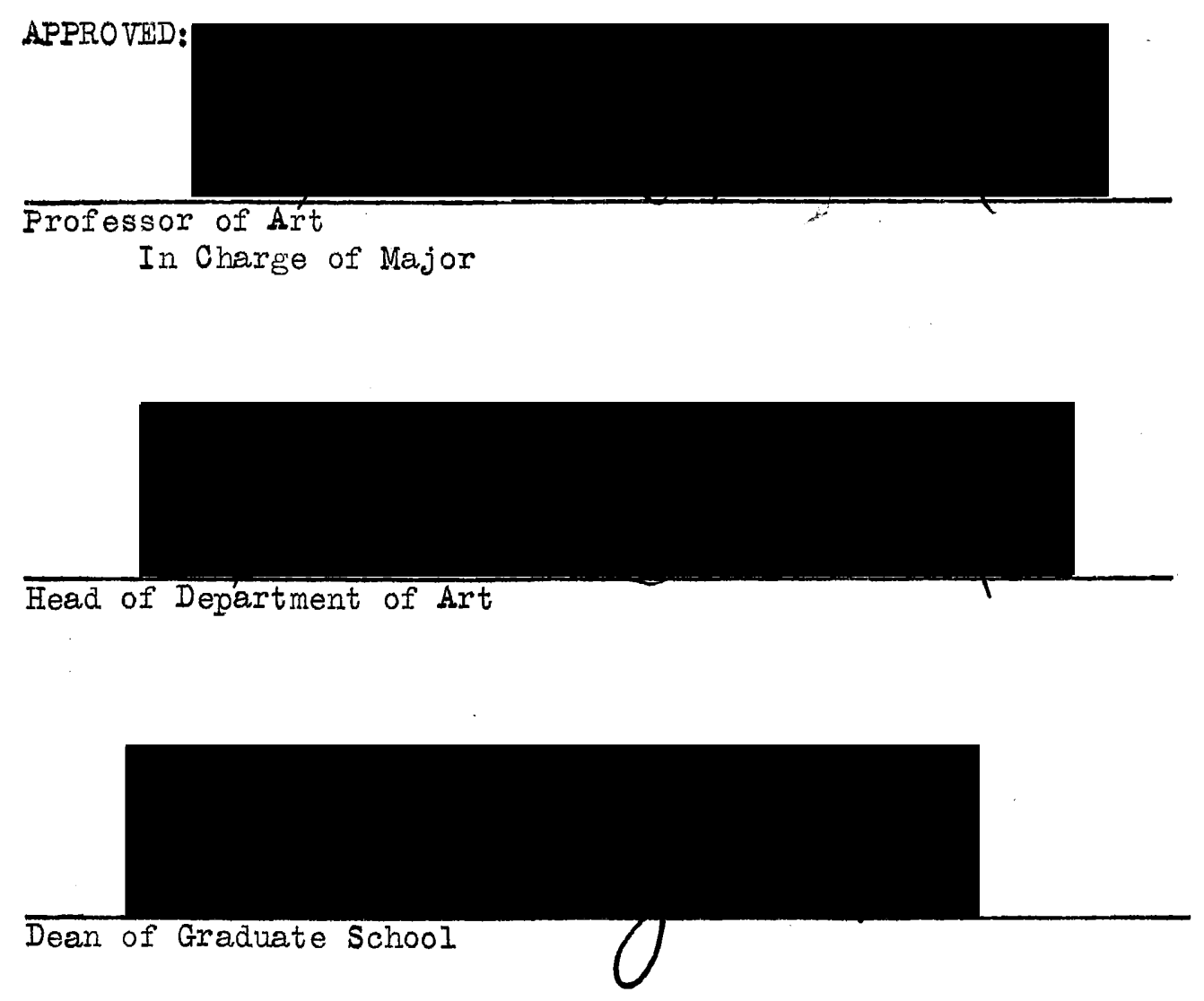

Date this is presented August 1, 1967

Typed by Helen Stratikos for Perry Theo Carandanis 
IANDSCAPE AND FIGURE COMPOSITION

IN REILATION TO SPACE, COLOR, AND IINE

by

\title{
PERRY THEO CARANDANIS
}

\author{
A THESIS \\ submitted to \\ PORTLAIND STATT COLIEGE
}

in partial fulfillment of

the requirements for the

degree of

Master of Science in Teaching

August 1967 
TABLE OF CONTENTS

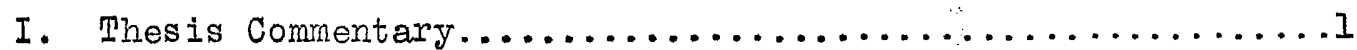

II. Thesis Paintings

Painting (Figure Composition)..............

Painting \#2 (Figure Composition)..............

Painting $\frac{\|}{\pi 3}$ (Figure Composition)..............

Painting \#4 (Figure Composition)...............

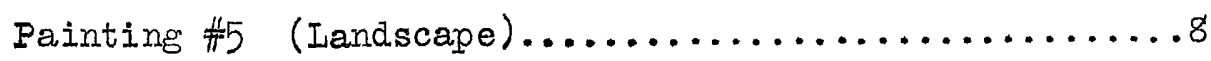

Painting \#6 (Landscape) $\ldots \ldots \ldots \ldots \ldots \ldots \ldots \ldots \ldots$

Painting \#7 (Landscape).................... 


\section{IANDSCAPI AND FIGURE COMPOSITION IN RHIATION TO SPACE, COIOR AND IINT}

The media for this thesis are acrylic polymers painted on upsom board. Acrylic was selected rather than oil because of its unique ability to set and dry quickly, the ease with which it applies to the surface, and the fact that it produces no harmful vapors. It is almost identical to oil paint in appearance, yet it can be mixed with water. It can also be made into a thick impasto or diluted into a transparent wash. It is an ideal medium, and easily adaptable to the objectives.

The painting surface is upsom board, a compressed cardboard, and is used because it is easily cut and sized within minutes. Some artists object to its use because it does not offer the sensitive resilience to the brush that canvas might, however, this is not disturbing since my feeling seems to be completely in the manipulation of the brush. The pebbled surface of the board approximates the qualities of a canvas surface, and the board also has the advantage of not warping as many poorly stretched canvases do.

Prints from these transparencies were not made because of the several comments received of the poor reproductive qualities. Anyone interested in examining this thesis work will have to sacrifice convenience for quality.

Painting \#I is basically a composition of three figures in which the motif of the bust becomes a dominant characteristic to remain 
throughout the entire sequence of paintings. The painting has a strong vertical structure of masses alternating from dark to light, the dark areas becoming cooler and lighter, and light areas warmer and luminous. The light lines play primarily upon the dark areas and the dark lines on the light areas. The masses of verticals interplay in color so that optical movement from one to the other is modified. The red of the table is held back by its color intensity and interspersed relationships with other colors, but remains as a vital part of the painting.

In Painting $\frac{\|}{t 2}$, the line becomes suddenly more structural against strong, tightly composed verticals and the painting moves forward on the surface into an almost flattened, decorative pattern. The bust motif becomes cubical in the sense that the painting is more formally arranged into modules of color, and there exists a predominance of warm hues against one cool vertical passage on the right.

The organization of color of Painting. \#3 has changed considerably in that the neutral, warm passages have been pushed out to the extreme sides, allowing a large area of cool to prevail throughout the center. The composition is beconing even more formal as the structure ljes in the two large blue symetrical cross-forms. The modular groupings are still. apparent as the bust motif retains its sense of formality in an alternating sequence of value changes from the left of the painting to the right. The original line motif that evolved into a new importance 
- of cubical shape has lost its importance as line and a new kind of caligraphic line develops and find more maturity in Painting 44.

Painting 144 has broken from the perpendicular structure into a relationship of diagonals and there becomes a developing interest in the caligraphic movement of line. Space is becoming rather open as forms transcend into mass and the composition noves more readily from left to right as the light and medium planes of color move in conjunction. In this painting there is a sense of delicacy existing because of the new sensitivity to line, the general high key of the painting, the introduction of the fresh greens, the new sense of random openness of volume, and the consistent close relationships of values. The figures are also becoming more apparent as entities of form rather than formlessness.

Painting \#5 assumes a horizontal rather than vertical movement, and the masses of color and line delineation of the motif suddenly become one of earth forms. The colors remain primarily cool and space takes on a feeling of concrete existence. The painting has lost any sense of decorativeness or delicacy of touch and the movement in space remains restricted in which the planes become interposed, weaving, and less analytical.

In Painting $\# 6$ color is intentionally eliminated and gives emphasis to the elements of line and space, however, total elimination of color could not be resolved. The singular, super-imposed line, has 
taken on another characteristic in which it becomes sketchy and vibrating and produces a kind of tension indigenous to natural forms. The movements of light and dark are also becoming thrustlike rather than in the formally placed figure cormositions. Some symbolic elements of nature also begin to appear, possibly because of the conscious deletion of imagery. The openness of form elicits a feeling of magnitude and directness in which the brush strokes are bold, harshly placed, and are pushing in unison. Force seems to be replacing form. However, with all the dynamic and violent movement, the basic composition's formality is still retained in which the main thrust of dark comes through the center of the painting and severs the light areas into almost equal proportions.

In Painting $\# 7$ the introduction of the sky planes merge with the earth to form a perfect harmonious interplay of color, line, space and landscape. The hue intensity of blues, oranges and yellow-greens, is vibrant and forceful, and line is introduced to give significance to form but does not dominate or super-impose.

In examining the total thesis work, space seems to become an increasingly important factor. Iarge areas of hue or value groupings have to retain a sense of structure so that the composition will aesthetically hold together. In the earlier paintings it became more apparent that it was necessary to consider this kind of structure. Consequently, there developed four or five dominant vertical movements which eventually subsided. 
Color has proved to be a vital element in the total expressive quality of the works and is the primary catalyst for reactions upon the other elements. Color is not approached from a standpoint of methodology but is applied intuitively, the only manner in which the greatest sense of spontaneity could be achieved! Major color areas and tonal ranges are preconceived and analyzed but once the painting commences any color relationships are subject to change depending on the existing emotional state. Color theory is utilized but in application it is reduced to primarily the subconscious levels.

The linear notif which has provided exciting challenges, is the short, swift line that weaves in and out of the painting, and becomes sometimes more and sometimes less concentrated. It appears as a line or at times a shape, and usually plays an equal role in negative and positive spaces. It exists much of the time as an undefinable shape but sometimes delineates recognizable shapes. It can be described as a form of energy. The motif developed as a result of a direct method of applying the paint to the surface with a brush. Much of the time it composes the main body of the work but on occasion becomes super-imposed.

The approach to the thesis paintings, as surmised, is direct and subjective. The mind, eyes and hand work as one. It is difficult to say which of the three plays a more dominant role but the saccess appears to depend on the three working in equal proportions. There also seems to be a development in the preoccupation with the emotional 
forces and stresses that are manifested in the painting, and in the search for new visual experiences there is a hindrance in having to work within the framework of recognizable objective forms that dictate too readily the space within trite dimensions. Once an independence from imagery is achieved the freedom exists to plunge areas of painting into deep space or pull volume out of flat areas at will, or activate an area with whatever color, shape, or line is appropriate at the time. An entire passage can be removed or reworked into a completely new creative statement. In this series of thesis paintings there has developed a new level of freshness, vitality and enrichment that did not previously exist in my other works.

None of the works are completely emotional statements from the beginning of the paintings to the end. The tempo of work is occasionally slowed and periodically, long analytical considerations are made. The groundwork is a contrived spontaneity, that is, the brush strokes are laid down after much deliberation, but still retain as much as possible the freshness and freedom of the unpremeditated quality.

At times there is a need to return to a realistic rendering of subject matter mainly as a reinforcement in understanding volumetric forms. These studies are of figures or landscapes sketched with pencil or charcoal on paper prior to beginning a painting and sometimes several preliminary compositional sketches are made when the need is felt. 
In these thesis works, many significant discoveries of personal enrichment could hardly be verbally translated. One would have to duplicate the actual mental and physical processes in order to become completely enlightened with the same experiences. It is my hope that one who examines and ponders this work will see with each sitting new revelations and hidden subtleties emerge, and to virtually undergo the same satisfying excitenent which $I$ had in creating the work. An important factor to bear in mind is not the technical knowledge in this type of painting, but rather the thoughts that transpire in the artist's nind at the moment the brush touches the canvas.

As one constantly searches for aesthetic answers he will discover that it is an endless search and that he must not cling too strongly to traditional beliefs and stereotyped ideas, but should be flexible enough to accept fresh and original thought, which is of course the basis for creativity, and to accept it not as a break from traditional dogmas but as an extension into a new scope of thinking. 


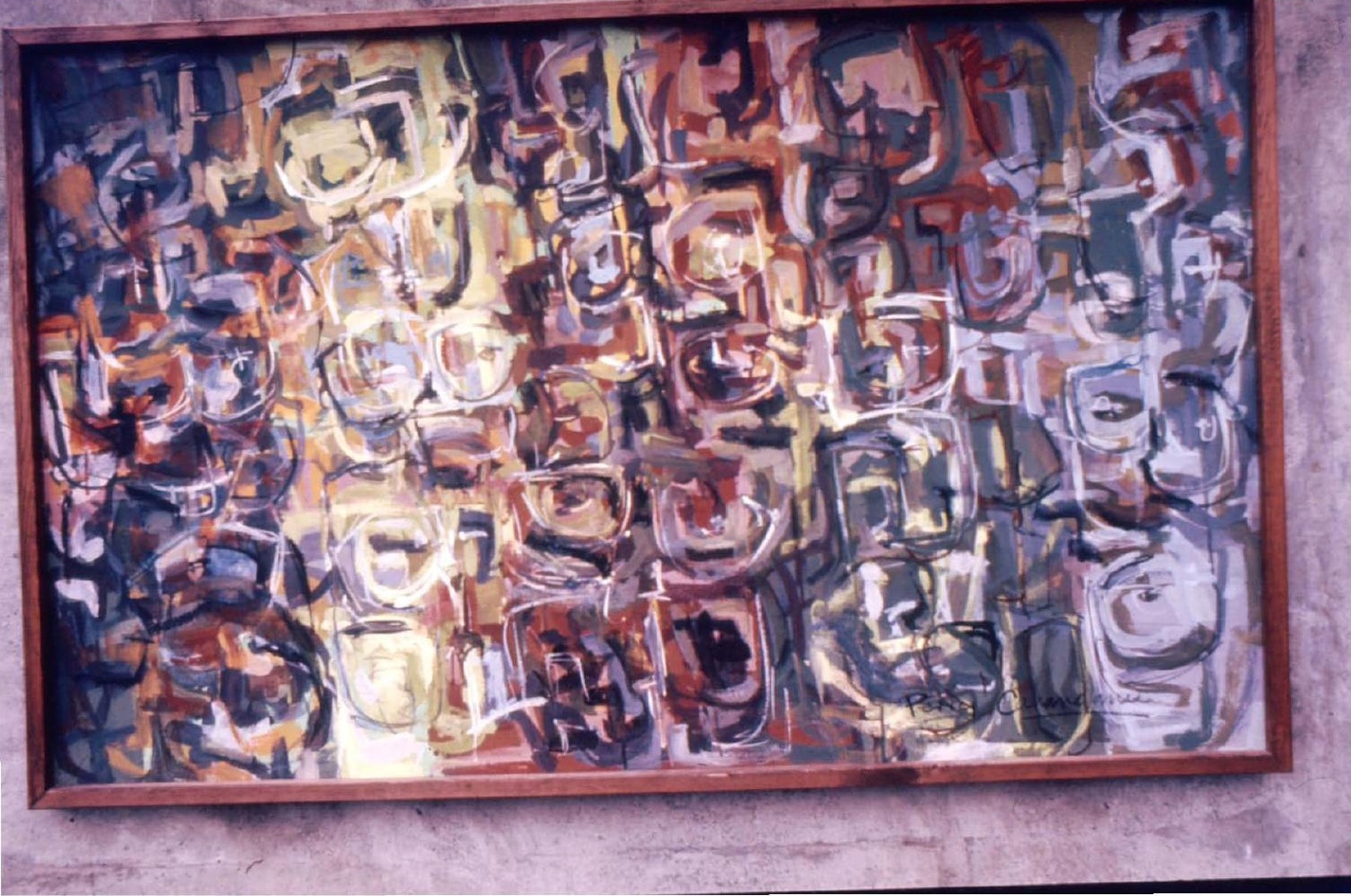




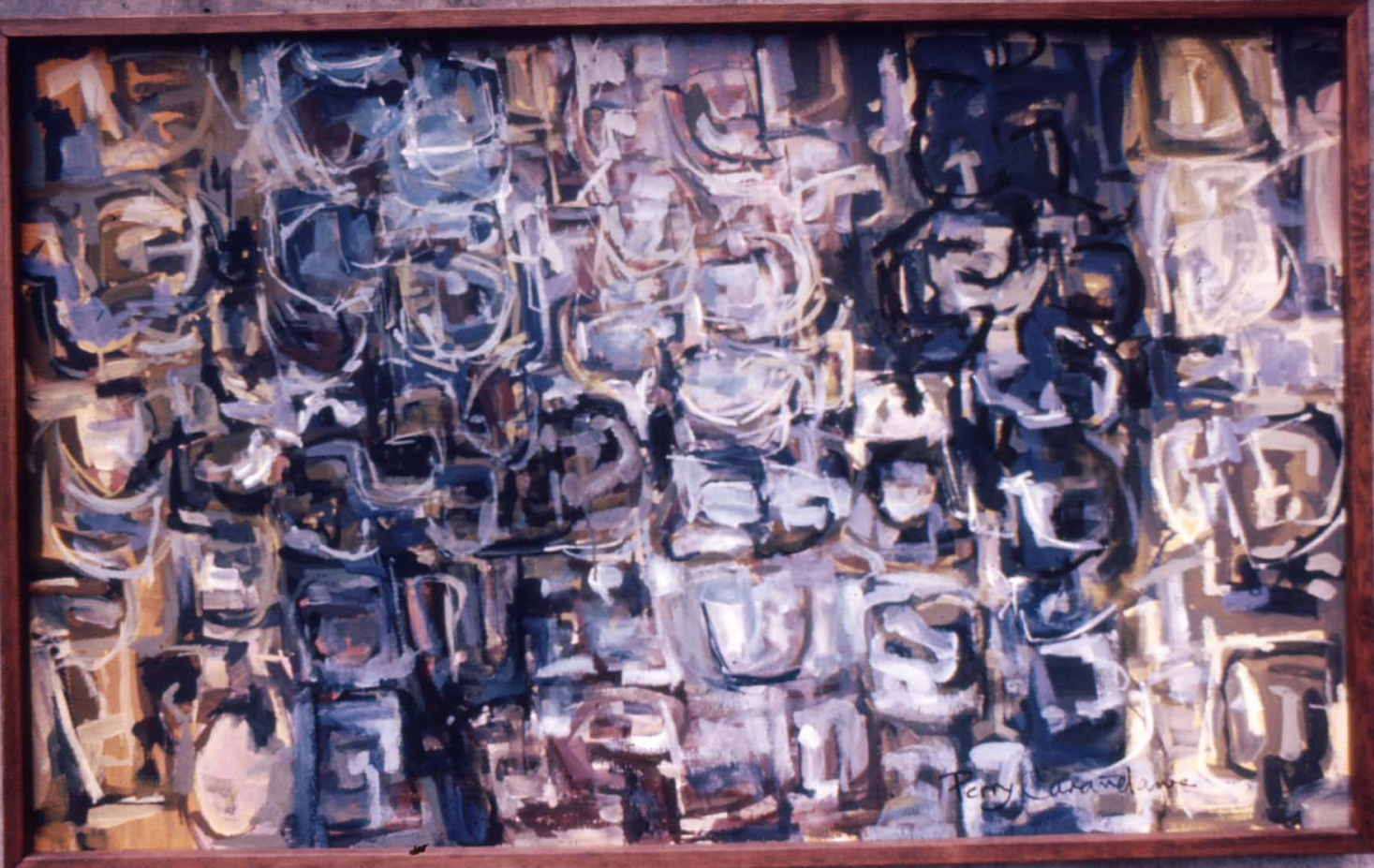




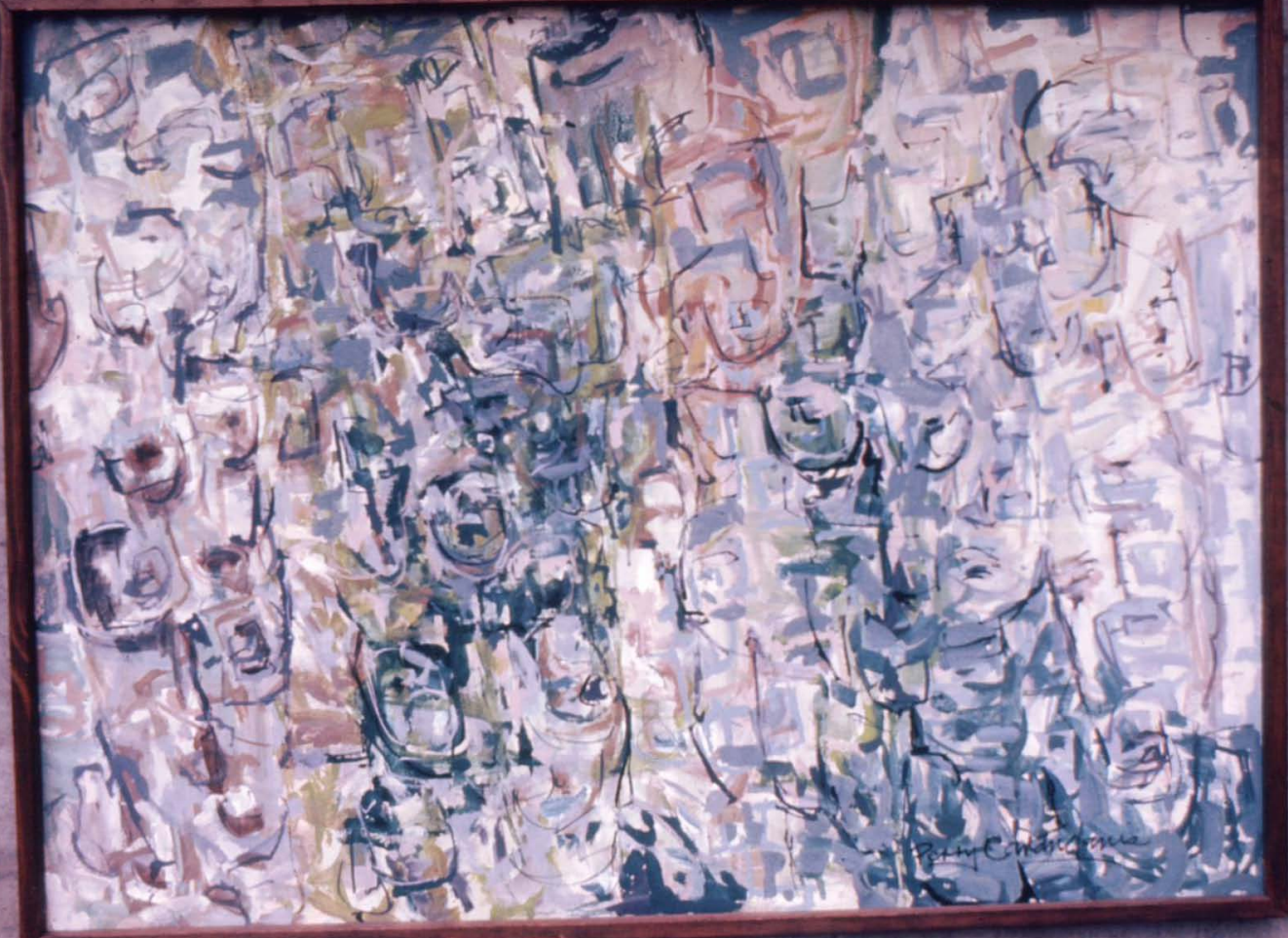




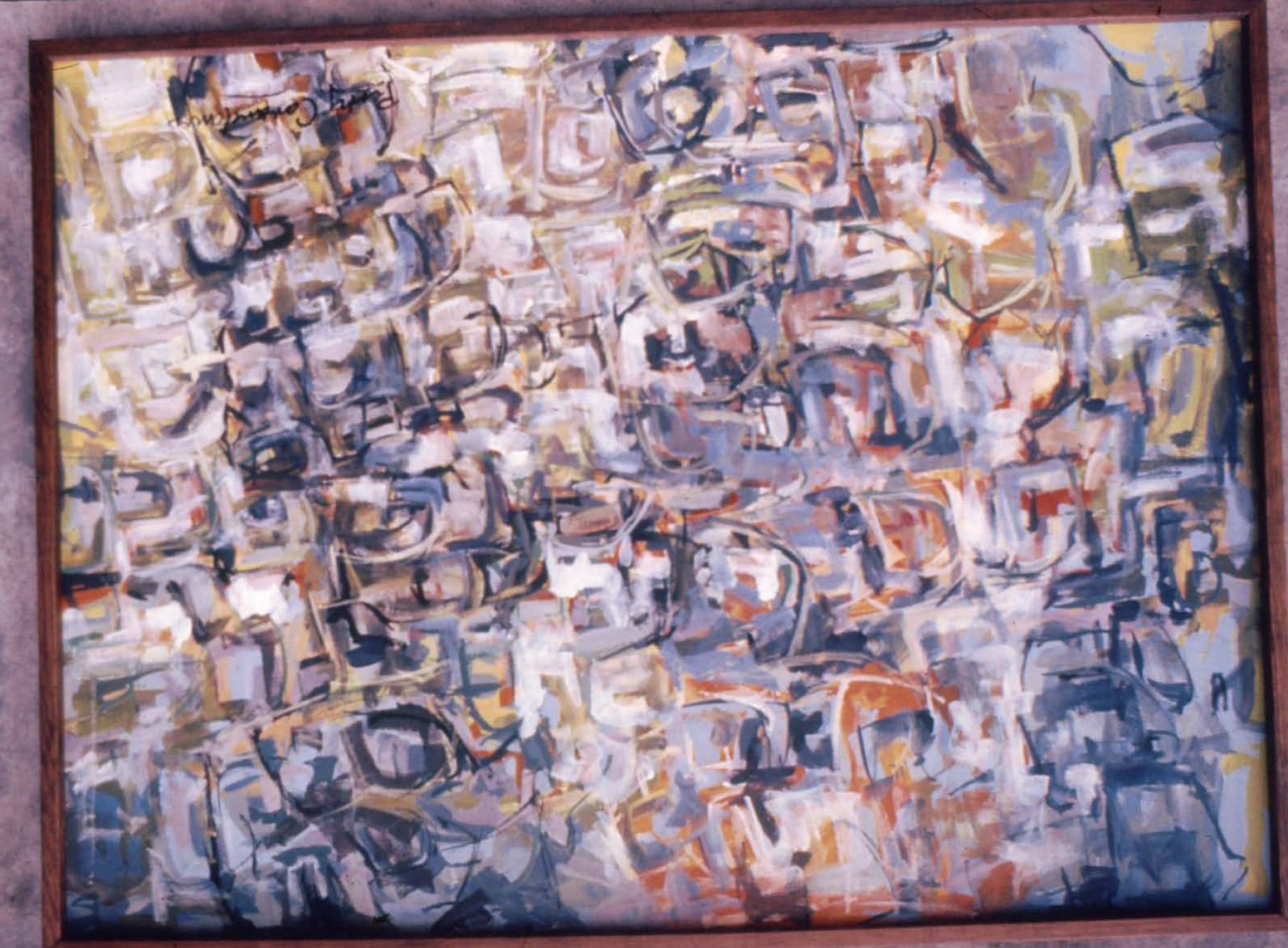




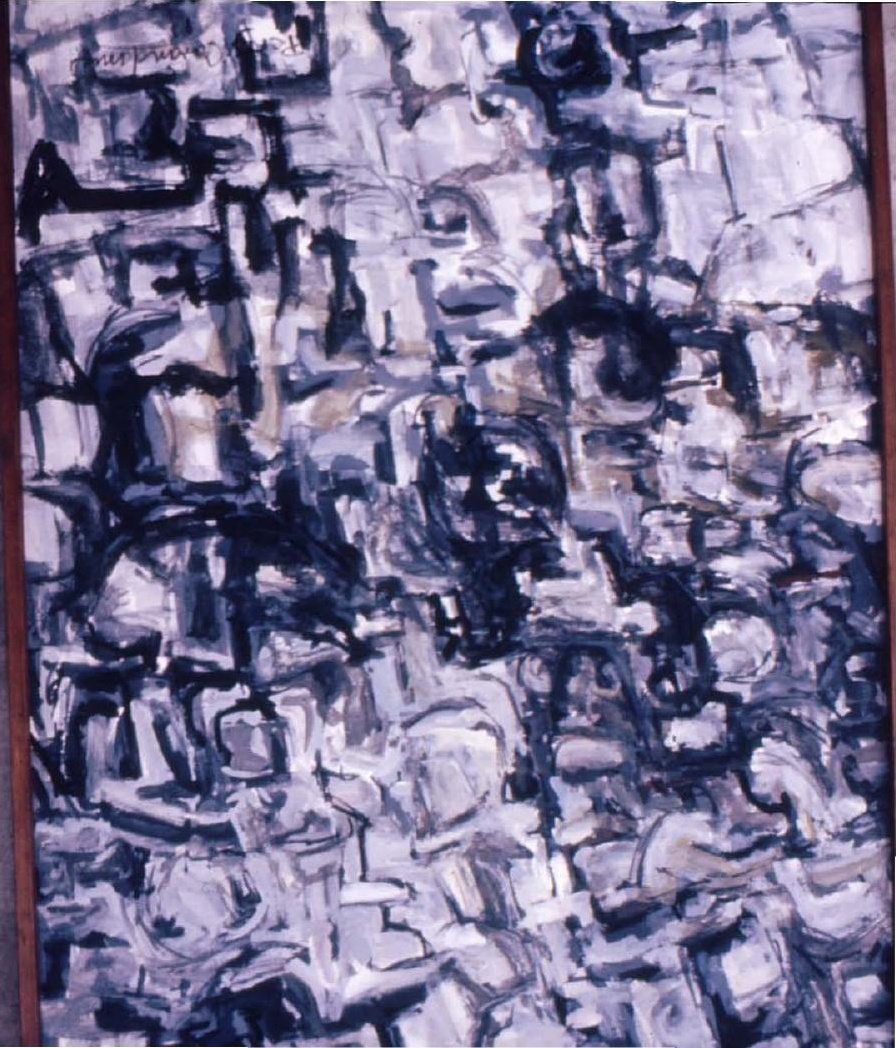




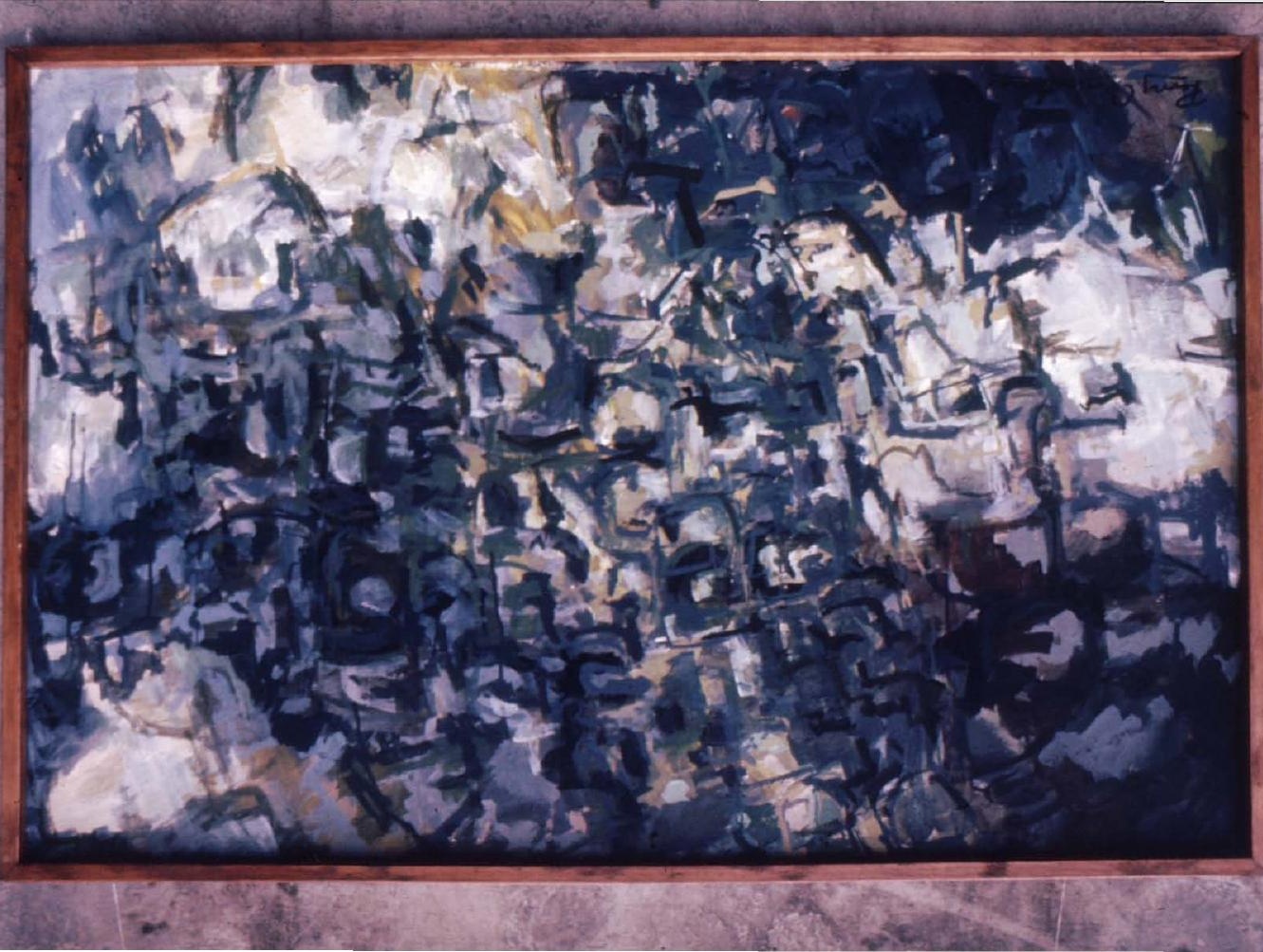

